\title{
Sharp threshold for blow-up and global existence in a semilinear parabolic equation with variable source
}

Jinge Yang ${ }^{*}$ and Haixiong Yu

\section{"Correspondence:}

jgyang2007@yeah.net

School of Sciences, Nanchang

Institute of Technology, Nanchang,

330099, P.R. China

\begin{abstract}
This paper deals with a semilinear parabolic equation with variable source under the case that the initial energy is less than the potential well depth. We deduce a sharp threshold for blow-up and global existence of solutions. Furthermore, we conclude that the global solution decays as the time goes to infinity.
\end{abstract}

Keywords: semilinear parabolic equations; variable source; initial energy; global existence; blow-up

\section{Introduction}

In this paper, we consider an initial boundary value problem for the semilinear parabolic equation with variable exponent:

$$
\begin{cases}u_{t}=\Delta u+|u|^{p(x)-2} u, & x \in \Omega, t>0, \\ u(x, t)=0, & x \in \partial \Omega, t>0, \\ u(x, 0)=u_{0}(x), & x \in \Omega,\end{cases}
$$

where $\Omega$ is a bounded smooth domain of $\mathbb{R}^{N}(N \geq 3), u_{0} \in H_{0}^{1}(\Omega)$, and $p(x)$ is a continuous and bounded function satisfying

$$
2<p^{-}:=\inf _{x \in \Omega} p(x) \leq p^{+}:=\sup _{x \in \Omega} p(x)<2^{*}=\frac{2 N}{N-2} .
$$

Eq. (1.1) has been used to model a variety of important physical processes, such as electrorheological fluids (where $u$ is the velocity of moving fluids in electro-magnetic fields) [1], thermo-rheological flows or population dynamics [2,3]. There is a substantial amount of work concerning the case $p(x) \equiv p$, see, for example, [4-6].

To deal with the variable source, it is convenient to introduce a Lebesgue space $L^{p(\cdot)}(\Omega)$, defined as the space of measurable functions $u$ in $\Omega$ satisfying $\int_{\Omega}|u|^{p(x)} d x<\infty$. We mention that this kind of Lebesgue space or general Sobolev space with variable exponent and their applications have got a lot of attention, see the monograph [7] and some recent work [8-11] for instance.

\section{Springer}


With the norm

$$
\|u\|_{p(\cdot)}:=\|u\|_{L^{p(\cdot)}}(\Omega)=\inf \left\{\lambda>0: \int_{\Omega}\left|\frac{u}{\lambda}\right|^{p(x)} d x \leq 1\right\}
$$

the space $L^{p(\cdot)}(\Omega)$ is a Banach space and

$$
\inf \left\{\|u\|_{p(\cdot)}^{p^{+}},\|u\|_{p(\cdot)}^{p^{-}}\right\} \leq \int_{\Omega}|u|^{p(x)} d x \leq \max \left\{\|u\|_{p(\cdot))}^{p^{+}},\|u\|_{p(\cdot)}^{p^{-}}\right\},
$$

see [12]. Combining Corollary 3.34 in [12] and the Poincaré inequality, we have

$$
\|u\|_{p(\cdot)} \leq B\|\nabla u\|_{2}
$$

Regarding variable sources, Pinasco [13] proved that the solution of (1.1) blows up in finite time provided that $p^{-}>1$ and the initial data is large enough. This result was then extended to $p^{+}>1$ by Ferreira et al. in [14]. For some positive initial energy, Wu et al. [15] gave a blow-up condition.

Proposition 1.1 (Theorem 1.1 in [15]) Let

$$
E_{1}=: \frac{1}{p^{-}}\left(\frac{p^{+}-2}{2} B^{p^{+}} \alpha_{1}^{\frac{p^{+}}{2}}+\frac{p^{-}-2}{2} B^{p^{-}} \alpha_{1}^{\frac{p^{-}}{2}}\right),
$$

and

$$
\bar{E}_{1}=:\left(\frac{p^{+}-2}{p^{-}-2}\right)^{\frac{2}{p^{+}}}\left\{\frac{\alpha_{1}}{2}-\frac{1}{p^{-}}\left[B^{p^{+}}\left(\frac{p^{+}-2}{p^{-}-2}\right)^{\frac{p^{+}-2}{p^{+}}} \alpha_{1}^{\frac{p^{+}}{2}}+B^{p^{-}}\left(\frac{p^{+}-2}{p^{-}-2}\right)^{\frac{p^{-}-2}{p^{-}}} \alpha_{1}^{\frac{p^{-}}{2}}\right]\right\}<E_{1},
$$

where $\alpha_{1}$ is defined by

$$
\frac{1}{p^{-}}\left(B^{p^{+}} p^{+} \alpha_{1}^{\frac{p^{+}-2}{2}}+B^{p^{-}} p^{-} \alpha_{1}^{\frac{p^{-}-2}{2}}\right)=1
$$

Assume $1<\sqrt{2 p^{+}-1}<p^{-} \leq p^{+} \leq \frac{N+2}{N-2}$ and $0<E\left(u_{0}\right)<\bar{E}_{1}$. If $\left\|\nabla u_{0}\right\|_{2}^{2}>\alpha_{1}$, then the solution of Eq. (1.1) blows up in finite time.

Later, another blow-up condition was derived by Wang and He [16].

Proposition 1.2 (Theorem 1 in [16]) Assume $1<p^{-} \leq p^{+} \leq \frac{N+2}{N-2}$ and $0<E\left(u_{0}\right)<E_{2}=$ $\frac{p^{-}-2}{2 p^{-}} B_{1}^{-\frac{2 p^{-}}{p^{-}-2}}$ with $B_{1} \geq \max \{B, 1\}$. If $\left\|\nabla u_{0}\right\|_{2}^{2}>\alpha_{2}=B_{1}^{-\frac{2 p^{-}}{p^{-}-2}}$, then the solution of Eq. (1.1) blows up in finite time.

Motivated by the above research, in this paper we have the main purpose to look for a sharp threshold for blow-up and global existence of solutions of (1.1) in general case (1.2) and (1.7) (in Section 4, we show $E_{0}>\bar{E}_{1}$ and $E_{0} \geq E_{2}$ ). We mainly use the potential well method, which was used to study the case $p(x) \equiv p$ by Payne and Sattinger [17], and was widely used to consider other parabolic models during the last years, see, for example, [1821]. Similar to [22], local existence and uniqueness of solutions of (1.1) can be obtained by the Banach fixed point theorem as follows. 
Proposition 1.3 Assume that (1.2) holds. Then (1.1) admits a unique solution $u \in$ $C\left(\left[0, T_{\max }\right) ; H_{0}^{1}(\Omega)\right) \cap C^{1}\left(\left(0, T_{\max }\right) ; L^{2}(\Omega)\right)$, where $T_{\max }>0$ denotes the maximal existence time. Either $T_{\max }<+\infty$ and $\lim _{t \rightarrow T_{\max }}\|u\|_{H_{0}^{1}(\Omega)}^{2}=+\infty$ (we say that the solution blows up in finite time), or $T_{\max }=+\infty$ (we say that the solution is global in time).

Denote the energy functional

$$
E(u)=\int_{\Omega}\left[\frac{1}{2}|\nabla u(x, t)|^{2}-\frac{1}{p(x)}|u(x, t)|^{p(x)}\right] d x
$$

and the Nehari manifold

$$
\mathcal{N}=\left\{u \in H_{0}^{1}(\Omega) \mid N(u)=0, u \neq 0\right\}
$$

with

$$
N(u)=\left\langle E^{\prime}(u), u\right\rangle=\int_{\Omega}\left[|\nabla u(x, t)|^{2}-|u(x, t)|^{p(x)}\right] d x .
$$

In this paper, we assume that the initial energy is less than the potential well depth, namely

$$
E\left(u_{0}\right)<E_{0}:=\inf _{u \in \mathcal{N}} E(u)
$$

Now, we introduce our main results as follows.

Theorem 1.1 Assume that (1.2) and (1.7) hold. Then

(1) if $N\left(u_{0}\right)<0$, then the solution of Eq. (1.1) blows up in finite time;

(2) if $N\left(u_{0}\right) \geq 0$, then the solution $u$ of Eq. (1.1) is global in time and $u(t) \rightarrow 0$ strongly in $H_{0}^{1}(\Omega)$ as $t \rightarrow \infty$.

Finally, we consider applications of Theorem 1.1 and derive the following results.

Corollary 1.1 Assume (1.2) and $0<E\left(u_{0}\right)<\bar{E}_{1}$. The solution to Eq. (1.1) is global in time if $\left\|\nabla u_{0}\right\|_{2}^{2} \leq \alpha_{1}$.

Corollary 1.2 Assume (1.2) and $0<E\left(u_{0}\right)<E_{2}$. The solution of Eq. (1.1) is global in time if $\left\|\nabla u_{0}\right\|_{2}^{2} \leq \alpha_{2}$.

Remark 1.1 Combined with Proposition 1.1 and Proposition 1.2, the above corollaries imply that the blow-up conditions in [15] and [16] are also sharp there.

This paper is organized as follows. In Section 2, we determine the blow-up condition of solutions of Eq. (1.1). In Section 3, we deal with global existence condition and then conclude that the global solution decays as the time goes to infinity. In Section 4, we prove Corollaries 1.1 and 1.2. Finally, we summarize the main results of the current paper.

In the sequel, we use $\|\cdot\|_{p}$ to denote $L^{p}(\Omega)$ norm, and denote the inner product in $L^{2}(\Omega)$ by the symbol $(\cdot, \cdot)$. 


\section{Finite time blow-up}

In this section, we pay attention to studying blow-up of solutions to Eq. (1.1). To deal with Theorem 1.1(1), we first give some preliminary lemmas.

Lemma 2.1 It holds $E_{0}>0$.

Proof For $u \in \mathcal{N}$, that is $\int_{\Omega}|\nabla u|^{2} d x=\int_{\Omega}|u|^{p(x)} d x$, we have

$$
\int_{\Omega}|\nabla u|^{2} d x \leq \int_{\Omega}|u|^{p^{-}} d x+\int_{\Omega}|u|^{p^{+}} d x
$$

We then apply the Poincáre inequality to find that

$$
\begin{aligned}
\int_{\Omega}|\nabla u|^{2} d x & \leq \int_{\Omega}|u|^{p^{-}} d x+\int_{\Omega}|u|^{p^{+}} d x \\
& \leq C\left(\left(\int_{\Omega}|\nabla u|^{2} d x\right)^{\frac{p^{-}}{2}}+\left(\int_{\Omega}|\nabla u|^{2} d x\right)^{\frac{p^{+}}{2}}\right),
\end{aligned}
$$

thereby obtaining the inequality

$$
\left(\int_{\Omega}|\nabla u|^{2} d x\right)^{\frac{p^{-}}{2}-1}+\left(\int_{\Omega}|\nabla u|^{2} d x\right)^{\frac{p^{+}}{2}-1} \geq \frac{1}{C} .
$$

This implies

$$
\int_{\Omega}|\nabla u|^{2} d x \geq \min \left\{\left(\frac{1}{2 C}\right)^{\frac{2}{p^{-}-2}},\left(\frac{1}{2 C}\right)^{\frac{2}{p^{+}-2}}\right\} .
$$

Therefore, we deduce

$$
\begin{aligned}
E(u) & \geq \frac{1}{2} \int_{\Omega}|\nabla u|^{2} d x-\frac{1}{p^{-}} \int_{\Omega}|u|^{p(x)} d x \\
& =\left(\frac{1}{2}-\frac{1}{p^{-}}\right) \int_{\Omega}|\nabla u|^{2} d x+\frac{1}{p^{-}} N(u) \\
& \geq \frac{p^{-}-2}{2 p^{-}} \min \left\{\left(\frac{1}{2 C}\right)^{\frac{2}{p^{-}-2}},\left(\frac{1}{2 C}\right)^{\frac{2}{p^{+}-2}}\right\} .
\end{aligned}
$$

Due to the arbitrariness of $u \in \mathcal{N}$, we conclude $E_{0}>0$.

Lemma 2.2 Fix $u \in H_{0}^{1}(\Omega), u \neq 0$ and define $u^{\lambda}:=\lambda u$ with $\lambda>0$. Then

(1) There exists a unique positive constant $\lambda_{0}>0$ such that $u^{\lambda_{0}} \in \mathcal{N}$ and $E\left(u^{\lambda_{0}}\right)=\max _{\lambda>0} E\left(u^{\lambda}\right)$

(2) $\lambda_{0}<1$ if and only if $N(u)<0$;

(3) $\lambda_{0}=1$ if and only if $N(u)=0$.

Proof A direct computation yields

$$
\frac{d}{d \lambda} E\left(u^{\lambda}\right)=\lambda \int_{\Omega}|\nabla u|^{2} d x-\int_{\Omega} \lambda^{p(x)-1}|u|^{p(x)} d x=\frac{1}{\lambda} N(\lambda u),
$$


and

$$
\frac{d^{2}}{d \lambda^{2}} E\left(u^{\lambda}\right)=\int_{\Omega}|\nabla u|^{2} d x-\int_{\Omega}(p(x)-1) \lambda^{p(x)-2}|u|^{p(x)} d x .
$$

Set $f(\lambda):=\frac{d}{d \lambda} E(\lambda u)$. There exists a unique $\lambda_{1}>0$ such that

(i) $f\left(\lambda_{1}\right)=\max _{\lambda>0} f(\lambda)$,

(ii) $f(0)=0$,

(iii) $f(\lambda)$ increases in $\left(0, \lambda_{1}\right)$ and decreases in $\left(\lambda_{1},+\infty\right)$. Conclusions (1)-(3) are easy consequences of (i)-(iii).

Denote

$$
E^{\varepsilon}:=\inf \left\{E(u): N(u)=-\varepsilon, u \in H_{0}^{1}(\Omega)\right\}
$$

with $\varepsilon>0$.

Lemma 2.3 Let (1.2) hold. Then $E^{\varepsilon} \geq E_{0}-\frac{\varepsilon}{2}$.

Proof Choose a minimized sequence $\left(u_{i}\right) \subseteq H_{0}^{1}(\Omega)$ such that

$$
N\left(u_{i}\right)=-\varepsilon \quad \text { and } \quad E\left(u_{i}\right) \rightarrow E^{\varepsilon} \quad(\text { as } i \rightarrow \infty) .
$$

It follows from Lemma 2.2 that there exists a positive constant $\mu_{i} \in(0,1)$ such that

$$
N\left(\mu_{i} u_{i}\right)=0 .
$$

Therefore

$$
\begin{aligned}
E_{0} \leq E\left(\mu_{i} u_{i}\right) & =\frac{1}{2} N\left(\mu_{i} u_{i}\right)+\int_{\Omega}\left(\frac{1}{2}-\frac{1}{p(x)}\right)\left|\mu_{i} u_{i}(x, t)\right|^{p(x)} d x \\
& =\int_{\Omega}\left(\frac{1}{2}-\frac{1}{p(x)}\right)\left|\mu_{i} u_{i}(x, t)\right|^{p(x)} d x \\
& \leq \int_{\Omega}\left(\frac{1}{2}-\frac{1}{p(x)}\right)\left|u_{i}(x, t)\right|^{p(x)} d x \\
& =E\left(u_{i}\right)-\frac{1}{2} N\left(u_{i}\right) \\
& =E\left(u_{i}\right)+\frac{\varepsilon}{2} .
\end{aligned}
$$

Let $i \rightarrow \infty$, then $E_{0} \leq E^{\varepsilon}+\frac{\varepsilon}{2}$.

Lemma 2.4 (Lemma $2.1[15]) \frac{d}{d t} E(u)=-\int_{\Omega} u_{t}^{2} d x \leq 0$.

Now we can prove Theorem 1.1(1) via the potential well method [17] and the Kaplan method [23].

Proof of Theorem 1.1(1) By Lemma 2.4, we have $E(u) \leq E\left(u_{0}\right)<E_{0}$. We claim that the solution $u$ blows up in finite time provided that $N\left(u_{0}\right)<0$. Otherwise, assume that $u$ is global 
in time. Choose $\varepsilon=\min \left\{E_{0}-E\left(u_{0}\right),-N\left(u_{0}\right)\right\} / 2$. Then $N\left(u_{0}\right) \leq-2 \varepsilon<-\varepsilon, E\left(u_{0}\right) \leq E_{0}-2 \varepsilon<$ $E_{0}-\frac{\varepsilon}{2} \leq E^{\varepsilon}$ by Lemma 2.3. Consequently, $N(u)<-\varepsilon$ for all $t>0$. Indeed, if there exists $t_{0}>0$ such that $N\left(u\left(t_{0}\right)\right)=-\varepsilon$, then $E\left(u\left(t_{0}\right)\right) \geq E^{\varepsilon}$ by the definition of $E^{\varepsilon}$. This is impossible since $E\left(u_{0}\right)<E^{\varepsilon}$ and Lemma 2.4.

Let $M(t)=\frac{1}{2} \int_{\Omega}|u(x, t)|^{2} d x$. Then by (1.1) we get that

$$
\begin{aligned}
M^{\prime}(t) & =\int_{\Omega} u(x, t) u_{t}(x, t) d x \\
& =-\int_{\Omega}|\nabla u(x, t)|^{2} d x+\int_{\Omega}|u(x, t)|^{p(x)} d x \\
& =-N(u)>\varepsilon .
\end{aligned}
$$

Consequently, we have

$$
\lim _{t \rightarrow \infty} M(t)=+\infty
$$

Next, we derive a contradiction by showing that $M(t)$ blows up in finite time. We deal with variable source as

$$
\begin{aligned}
\int_{\Omega}|u|^{p(x)} d x & =\int_{|u| \leq 1}|u|^{p(x)} d x+\int_{|u| \geq 1}|u|^{p(x)} d x \\
& \geq \int_{|u| \leq 1}|u|^{p^{+}} d x+\int_{|u| \geq 1}|u|^{p^{-}} d x \\
& \geq \int_{|u| \geq 1}|u|^{p^{-}} d x \\
& =\int_{|u| \leq 1} d x+\int_{|u| \geq 1}|u|^{p^{-}} d x-|\Omega| \\
& \geq \int_{|u| \leq 1}|u|^{p^{-}} d x+\int_{|u| \geq 1}|u|^{p^{-}} d x-|\Omega| \\
& \geq \int_{\Omega}|u|^{p^{-}} d x-|\Omega|,
\end{aligned}
$$

where $|\Omega|$ is the measure of $\Omega$. By the Hölder inequality, we have

$$
\begin{aligned}
\int_{\Omega}|u|^{p(x)} d x & \geq|\Omega|^{\frac{2-p^{-}}{2}}\left(\int_{\Omega}|u|^{2} d x\right)^{\frac{p^{-}}{2}}-|\Omega| \\
& =|\Omega|^{\frac{2-p^{-}}{2}} M(t)^{\frac{p^{-}}{2}}-|\Omega| .
\end{aligned}
$$

Therefore, by Lemma 2.4, we rewrite $M^{\prime}(t)$ in (2.1) as

$$
\begin{aligned}
M^{\prime}(t) & =-2 E(u)+\int_{\Omega}\left(1-\frac{2}{p(x)}\right)|u|^{p(x)} d x \\
& \geq-2 E\left(u_{0}\right)+\left(1-\frac{2}{p^{-}}\right) \int_{\Omega}|u|^{p(x)} d x \\
& \geq\left(1-\frac{2}{p^{-}}\right)|\Omega|^{\frac{2 p^{-}}{2}} M(t)^{\frac{p^{-}}{2}}-\left(1-\frac{2}{p^{-}}\right)|\Omega|-2 E\left(u_{0}\right) .
\end{aligned}
$$


It follows from (2.2) that there exists $t_{0}>0$ such that

$$
\left(1-\frac{2}{p^{-}}\right)|\Omega|^{\frac{2-p^{-}}{2}} M(t)^{\frac{p^{-}}{2}} \geq 2\left[\left(1-\frac{2}{p^{-}}\right)|\Omega|+2 E\left(u_{0}\right)\right]
$$

for $t \geq t_{0}$. Thus $M^{\prime}(t) \geq \frac{1}{2}\left(1-\frac{2}{p^{-}}\right)|\Omega|^{\frac{2-p^{-}}{2}} M(t)^{\frac{p^{-}}{2}}$. This immediately implies that $M(t)$ blows up in finite time, a contradiction.

\section{Global existence of solutions}

In this section, we consider global existence of solutions to Eq. (1.1) and study asymptotic behavior of the global solution.

Proof of Theorem 1.1(2) We divide the proof into two cases: (i) $N\left(u_{0}\right)=0$, (ii) $N\left(u_{0}\right)>0$. If $N\left(u_{0}\right)=0$, then $u_{0}=0$. Otherwise, if $u_{0} \neq 0$, then $E\left(u_{0}\right) \geq E_{0}$. This is a contradiction to the assumption condition (1.7). By uniqueness of solutions to (1.1), we have the solution $u(t)=0$ for all $t \geq 0$.

If $N\left(u_{0}\right)>0$, if $u(t)=0$ for some $t$, then $u(s)=0$ for $s \geq t$ by uniqueness, and the conclusion is true. Hereafter, we assume that $u(t) \neq 0$ for all $t \in(0, T)$. We claim that $N(u)>0$ as long as the solution $u$ exists. Otherwise, there exists $t_{0}$ such that $N\left(u\left(t_{0}\right)\right)=0$. By Lemma 2.4, $E\left(u\left(t_{0}\right)\right) \leq E\left(u_{0}\right)<E_{0}$, which contradicts the definition of $E_{0}$. Therefore,

$$
\begin{aligned}
E_{0}>E(u) & \geq \frac{1}{2} \int_{\Omega}|\nabla u|^{2} d x-\int_{\Omega} \frac{1}{p^{-}}|u|^{p(x)} d x \\
& =\left(\frac{1}{2}-\frac{1}{p^{-}}\right) \int_{\Omega}|\nabla u|^{2} d x+\frac{1}{p^{-}} N(u)>\left(\frac{1}{2}-\frac{1}{p^{-}}\right) \int_{\Omega}|\nabla u|^{2} d x .
\end{aligned}
$$

This with the Poincaré inequality yield that $\|u\|_{H_{0}^{1}(\Omega)}$ is uniformly bounded. By Proposition 1.3, there exists a global solution of Eq. (1.1).

Next, we investigate long time behavior of the global solution to Eq. (1.1). Basing on the above process, if $N\left(u_{0}\right)=0$, then $u(t)=0$ for all $t \geq 0$. The conclusion is true. If $N\left(u_{0}\right)>0$, then the global solution $u$ is uniformly bounded in $H_{0}^{1}(\Omega)$ and $N(u) \geq 0$. Consequently, $E(u) \geq 0$. Noting the equality

$$
\int_{0}^{t} \int_{\Omega} u_{t}^{2} d x d s+E(u)=E\left(u_{0}\right)
$$

by (1.1), we have

$$
\int_{0}^{\infty} \int_{\Omega} u_{t}^{2} d x d s \leq E\left(u_{0}\right)
$$

Therefore, there exists a time sequence $\left\{t_{i}\right\}_{i=1}^{\infty}$ with $t_{i} \rightarrow \infty$, as $i \rightarrow \infty$, such that $\left\|u_{t}\left(t_{i}\right)\right\|_{2}^{2} \rightarrow 0$. Since $u\left(t_{i}\right)$ is bounded in $H_{0}^{1}(\Omega)$ and thus $|u|^{p(\cdot)-2} u\left(t_{i}\right)$ is bounded in $H^{-1}(\Omega)$, going to a subsequence if necessary, still denoted by $u\left(t_{i}\right)$,

$$
\begin{aligned}
& u\left(t_{i}\right) \rightarrow \varphi \quad \text { weakly in } H_{0}^{1}(\Omega), \\
& u\left(t_{i}\right) \rightarrow \varphi \quad \text { strongly in } L^{q}(\Omega) \quad\left(2 \leq q<2^{*}\right), \\
& \left|u\left(t_{i}\right)\right|^{p(\cdot)-2} u\left(t_{i}\right) \rightarrow|\varphi|^{p(\cdot)-2} \varphi \quad \text { weakly in } H^{-1}(\Omega) .
\end{aligned}
$$


Multiplying (1.1) by $v \in H_{0}^{1}(\Omega)$ and integrating, we have

$$
\left(u_{t}\left(t_{i}\right), v\right)+\left(\nabla u\left(t_{i}\right), \nabla v\right)=\left\langle|u|^{p(\cdot)-2} u\left(t_{i}\right), v\right\rangle .
$$

Letting $i \rightarrow \infty$ in the above equality, we obtain that

$$
(\nabla \varphi, \nabla v)=\left\langle|\varphi|^{p(\cdot)-2} \varphi, v\right\rangle .
$$

Choosing $v=\varphi$ in the above equality, we have $N(\varphi)=0$. By (3.2) and the mean value theorem, we derive that

$$
\lim _{i \rightarrow \infty} \int_{\Omega}\left|u\left(t_{i}\right)\right|^{p(x)} d x=\int_{\Omega}|\varphi|^{p(x)} d x .
$$

By the weak semi-continuity of $H_{0}^{1}(\Omega)$ norm and (3.5), we have

$$
E(\varphi) \leq \liminf _{i \rightarrow \infty} E\left(u\left(t_{i}\right)\right)<E_{0} .
$$

This with $N(\varphi)=0$ yield that $\varphi=0$. Setting $v=u\left(t_{i}\right)$ in (3.4), observing that

$$
\left|\left(u_{t}\left(t_{i}\right), u\left(t_{i}\right)\right)\right| \leq\left\|u_{t}\left(t_{i}\right)\right\|_{2}\left\|u\left(t_{i}\right)\right\|_{2} \rightarrow 0
$$

we get that

$$
\lim _{i \rightarrow \infty}\left\|\nabla u\left(t_{i}\right)\right\|_{2}^{2}-\int_{\Omega}\left|u\left(t_{i}\right)\right|^{p(x)} d x=0 .
$$

This with (3.5) imply that

$$
\lim _{i \rightarrow \infty}\left\|\nabla u\left(t_{i}\right)\right\|_{2}^{2}=\lim _{i \rightarrow \infty} \int_{\Omega}\left|u\left(t_{i}\right)\right|^{p(x)} d x=\int_{\Omega}|\varphi|^{p(x)} d x=0 .
$$

Consequently, $E\left(u\left(t_{i}\right)\right) \rightarrow 0$ as $i \rightarrow \infty$. This with the fact that $E(u(t))$ is decreasing with respect of time $t$ and $E(u(t)) \geq 0$ imply that $\lim _{t \rightarrow \infty} E(u(t))=0$.

Rewrite $E(u(t))$ as

$$
E(u(t))=\frac{1}{2} N(u(t))+\int_{\Omega}\left(\frac{1}{2}-\frac{1}{p(x)}\right)|u(t)|^{p(x)} d x .
$$

It follows from $N(u(t)) \geq 0$ that

$$
\begin{aligned}
\int_{\Omega}|u(t)|^{p(x)} d x & \leq \frac{2 p^{-}}{p^{-}-2} \int_{\Omega}\left(\frac{1}{2}-\frac{1}{p(x)}\right)|u(t)|^{p(x)} d x \\
& \leq \frac{2 p^{-}}{p^{-}-2} E(u(t)) \rightarrow 0, \quad \text { as } t \rightarrow \infty .
\end{aligned}
$$

Therefore,

$$
\|\nabla u(t)\|_{2}^{2}=2 E(u(t))+\int_{\Omega} \frac{2}{p(x)}|u(t)|^{p(x)} d x \rightarrow 0, \quad \text { as } t \rightarrow \infty .
$$

This with the Poincáre inequality imply that $u(t) \rightarrow 0$ strongly in $H_{0}^{1}(\Omega)$. 


\section{Proof of Corollaries $\mathbf{1 . 1}$ and $\mathbf{1 . 2}$}

To compare our results to those in $[15,16]$, we begin with analyzing $E_{0}$ defined in (1.7) more precisely.

\section{Lemma 4.1 Set}

$$
E_{3}:=\inf \left\{\max _{\lambda>0} E(\lambda u): u \in H_{0}^{1}(\Omega) \text {, and } u \neq 0\right\}
$$

It holds $E_{0}=E_{3}$.

Proof For any $u \in \mathcal{N}$, by Lemma 2.2, we have that

$$
E(u)=\max _{\lambda>0} E(\lambda u) \geq E_{3} .
$$

Therefore, $E_{0} \geq E_{3}$. On the other hand, for any $u \in H_{0}^{1}(\Omega), u(x) \neq 0$, by Lemma 2.2, there exists $\lambda_{0}>0$ such that $\max _{\lambda>0} E(\lambda u)=E\left(\lambda_{0} u\right)$ and $N\left(\lambda_{0} u\right)=0$. This implies that $\max _{\lambda>0} E(\lambda u) \geq E_{0}$. Because of the arbitrariness of $u$, we have $E_{3} \geq E_{0}$. The proof is complete.

Next, we compare $E_{0}$ with $\bar{E}_{1}$ and $E_{2}$, where $\bar{E}_{1}$ is defined in Proposition 1.1 and $E_{2}$ defined in Proposition 1.2, respectively.

Lemma 4.2 $E_{0}>\bar{E}_{1}$ and $E_{0} \geq E_{2}$.

Proof For any $u \in H_{0}^{1}(\Omega), u \neq 0$, define $u^{\lambda}=\lambda u$ with $\lambda>0$. It follows from (1.3) and the Poincaré inequality (1.4) that

$$
\begin{aligned}
E(\lambda u) & =E\left(u^{\lambda}\right) \\
& \geq \frac{1}{2}\left\|\nabla u^{\lambda}\right\|_{2}^{2}-\frac{1}{p^{-}} \int_{\Omega}\left|u^{\lambda}\right|^{p(x)} d x \\
& \geq \frac{1}{2}\left\|\nabla u^{\lambda}\right\|_{2}^{2}-\frac{1}{p^{-}}\left\|u^{\lambda}\right\|_{p(\cdot)}^{p^{-}}-\frac{1}{p^{-}}\left\|u^{\lambda}\right\|_{p(\cdot)}^{p^{+}} \\
& \geq \frac{1}{2}\left\|\nabla u^{\lambda}\right\|_{2}^{2}-\frac{1}{p^{-}} B^{p^{-}}\left\|\nabla u^{\lambda}\right\|_{2}^{p^{-}}-\frac{1}{p^{-}} B^{p^{+}}\left\|\nabla u^{\lambda}\right\|_{2}^{p^{+}} \\
& =: h\left(\left\|\nabla u^{\lambda}\right\|_{2}\right) .
\end{aligned}
$$

Choose $\lambda_{1}>0$ such that $\left\|\nabla u^{\lambda}\right\|_{2}=\alpha_{1}$, defined in (1.6), and $h\left(\left\|\nabla u^{\lambda_{1}}\right\|_{2}\right)=E_{1}$. Thus

$$
\max _{\lambda>0} E(\lambda u) \geq E\left(\lambda_{1} u\right) \geq h\left(\left\|\nabla u^{\lambda_{1}}\right\|_{2}\right)=E_{1}
$$

This with Lemma 4.1 yield that $E_{0} \geq E_{1}$. Since $E_{1}>\bar{E}_{1}$, we have $E_{0}>\bar{E}_{1}$.

Now we prove $E_{0} \geq E_{2}$. For any nontrivial function $u \in \mathcal{N}$, by (1.3), we have

$$
\begin{aligned}
\|\nabla u\|_{2}^{2} & =\int_{\Omega}|u|^{p(x)} d x \\
& \leq \max \left\{\|u\|_{p(\cdot)}^{p^{+}},\|u\|_{p(\cdot)}^{p^{-}}\right\} \leq \max \left\{B_{1}^{p^{+}}\|\nabla u\|_{2}^{p^{+}}, B_{1}^{p^{-}}\|\nabla u\|_{2}^{p^{-}}\right\} .
\end{aligned}
$$


Therefore, $\|\nabla u\|_{2} \geq B_{1}^{-\frac{p^{+}}{p^{+}-2}}$, or $\|\nabla u\|_{2} \geq B_{1}^{-\frac{p^{-}}{p^{-}-2}}$. As $B_{1}>1$ and $p^{+} \geq p^{-}$, we get $\|\nabla u\|_{2} \geq$ $B_{1}^{-\frac{p^{-}}{p^{-}-2}}$. Consequently,

$$
E(u) \geq\left(\frac{1}{2}-\frac{1}{p^{-}}\right)\|\nabla u\|_{2}^{2} \geq \frac{p^{-}-2}{2 p^{-}} B_{1}^{-\frac{2 p^{-}}{p^{-}-2}}=E_{2} .
$$

Due to the arbitrariness of $u \in \mathcal{N}$, we conclude $E_{0} \geq E_{2}$.

Finally, we prove Corollaries 1.1 and 1.2.

Proof of Corollary 1.1 Assume $\left\|\nabla u_{0}\right\|_{2}^{2} \leq \alpha_{1}$, where $\alpha_{1}$ is defined in (1.6). Then

$$
\frac{1}{p^{-}}\left(B^{p^{+}} p^{+}\left\|\nabla u_{0}\right\|_{2}^{p^{+}-2}+B^{p^{-}} p^{-}\left\|\nabla u_{0}\right\|_{2}^{p^{-}-2}\right) \leq 1 .
$$

Consequently,

$$
\begin{aligned}
\left\|\nabla u_{0}\right\|_{2}^{2} & \geq \frac{1}{p^{-}}\left(B^{p^{+}} p^{+}\left\|\nabla u_{0}\right\|_{2}^{p^{+}}+B^{p^{-}} p^{-}\left\|\nabla u_{0}\right\|_{2}^{p^{-}}\right) \\
& \geq B^{p^{+}}\left\|\nabla u_{0}\right\|_{2}^{p^{+}}+B^{p^{-}}\left\|\nabla u_{0}\right\|_{2}^{p^{-}} .
\end{aligned}
$$

By (1.3) and the Poincaré inequality (1.4), we have

$$
\begin{aligned}
\left\|\nabla u_{0}\right\|_{2}^{2} & \geq\left\|u_{0}\right\|_{p(\cdot)}^{p^{+}}+\left\|u_{0}\right\|_{p(\cdot)}^{p^{-}} \\
& \geq \int_{\Omega}\left|u_{0}\right|^{p(x)} d x,
\end{aligned}
$$

thereby we obtain $N\left(u_{0}\right) \geq 0$. Combining Lemma 4.2 and Theorem 1.1(2), we see that the solution of Eq. (1.1) is global.

Proof of Corollary 1.2 Since $\left\|\nabla u_{0}\right\|_{2}^{2} \leq \alpha_{2}=B_{1}^{-\frac{2 p^{-}}{p^{-}-2}}, B_{1} \geq 1$ and $p^{-} \leq p^{+}$, then $B_{1}^{-\frac{2 p^{-}}{p^{-}-2}} \geq$ $B_{1}^{-\frac{2 p^{+}}{p^{+}-2}}$ and

$$
\left\|\nabla u_{0}\right\|_{2}^{2} \leq \min \left\{B_{1}^{-\frac{2 p^{-}}{p^{-}-2}}, B_{1}^{-\frac{2 p^{+}}{p^{+}-2}}\right\}
$$

Consequently,

$$
\left\|\nabla u_{0}\right\|_{2}^{2} \geq \max \left\{B_{1}^{p^{-}}\left\|\nabla u_{0}\right\|_{2}^{p^{-}}, B_{1}^{p^{+}}\left\|\nabla u_{0}\right\|_{2}^{p^{+}}\right\} .
$$

Using (1.3) and the Poincaré inequality (1.4), we obtain that

$$
\begin{aligned}
\left\|\nabla u_{0}\right\|_{2}^{2} & \geq \max \left\{\left\|u_{0}\right\|_{p(\cdot)}^{p^{-}},\left\|u_{0}\right\|_{p(\cdot)}^{p^{+}}\right\} \\
& \geq \int_{\Omega}\left|u_{0}\right|^{p(x)} d x .
\end{aligned}
$$

This implies that $N\left(u_{0}\right) \geq 0$. The rest is the same as the proof of Corollary 1.1, and hence is omitted. 


\section{Conclusions}

The main aim of the current work is to study asymptotic behavior of solutions to the parabolic equation (1.1). We prove that, when the initial energy is smaller than the mountain pass level corresponding to the stationary equation of (1.1) (see (1.7) and Lemma 4.1), the initial Nehari functional plays an important role in determining asymptotic behavior of the solution of (1.1), see Theorem 1.1. That is, if the initial Nehari functional is negative, then the solution of (1.1) blows up in finite time, while there exists a global solution if the initial Nehari functional is nonnegative. Moreover, the global solution decays as the time goes to infinity. This result generalizes the ones in [15] and [16], see Lemma 4.2 for differences between them. As applications of Theorem 1.1, we derive two corollaries which yield that the blow-up conditions in [15] and [16] are also sharp there, see Corollaries 1.1, 1.2 and Remark 1.1.

\section{Competing interests}

The authors declare that they have no competing interests.

\section{Authors' contributions}

The authors declare that the manuscript was completed in cooperation with the same responsibility. All authors read and approved the final manuscript.

\section{Acknowledgements}

JY was partially supported by NSF of Jiangxi Province (GJJ161112), NNSF of China, No:61461032 and the Project of Nanchang Institute of Technology, No:2014KJ020. HY was partially supported by the STRP of Jiangxi Province, No:20151BAB211009.

\section{Publisher's Note}

Springer Nature remains neutral with regard to jurisdictional claims in published maps and institutional affiliations.

Received: 20 February 2017 Accepted: 18 May 2017 Published online: 30 May 2017

\section{References}

1. Acerbi, E, Mingione, G: Regularity results for stationary electrorheological fluids. Arch. Ration. Mech. Anal. 164, 213-259 (2002)

2. Antontsev, SN, Rodrigues, JF: On stationary thermo-rheological viscous flows. Ann. Univ. Ferrara, Sez. 7: Sci. Mat. 52, $19-36(2006)$

3. Pao, CV: Nonlinear Parabolic and Elliptic Equations. Plenum, New York (1992)

4. Deng, K, Levine, HA: The role of critical exponents in blow-up theorems: the sequel. J. Math. Anal. Appl. 243, 85-126 (2000)

5. Qi, YW: On the equation $u_{t}=\Delta u^{\alpha}+u^{\beta}$. Proc. R. Soc. Edinb., Sect. A 123, 373-390 (1993)

6. Quittner, P, Souplet, P: Superlinear Parabolic Problems: Blow-up, Global Existence and Steady States. Birkhäuser, Basel (2007)

7. Radulescu, V, Repovs, D: Partial Differential Equations with Variable Exponents: Variational Methods and Qualitative Analysis. Monographs and Research Notes in Mathematics. CRC Press, Boca Raton (2015)

8. Bisci, GM, Repovs, D: Multiple solutions for elliptic equations involving a general operator in divergence form. Ann. Acad. Sci. Fenn., Math. 39, 259-273 (2014)

9. Fu, Y, Shan, Y: On the removability of isolated singular points for elliptic equations involving variable exponent. Adv. Nonlinear Anal. 5, 121-132 (2016)

10. Mihailescu, M, Radulescu, V: Neumann problems associated to nonhomogeneous differential operators in Orlicz-Sobolev spaces. Ann. Inst. Fourier (Grenoble) 58, 2087-2111 (2008)

11. Mihailescu, M, Radulescu, V, Repovs, D: On a non-homogeneous eigenvalue problem involving a potential: an Orlicz-Sobolev space setting. J. Math. Pures Appl. 93, 132-148 (2010)

12. Diening, L, Harjulehto, P, Hästö, P, Rûžička, M: Lebesgue and Sobolev Spaces with Variable Exponents. Lecture Notes in Mathematics, vol. 2017. Springer, Heidelberg (2011)

13. Pinasco, JP: Blow-up for parabolic and hyperbolic problems with variable exponents. Nonlinear Anal. 71, 1049-1058 (2009)

14. Ferreira, R, Pablo, A, Pérez-Llanos, M, Rossi, JD: Critical exponents for a semilinear parabolic equation with variable reaction. Proc. R. Soc. Edinb., Sect. A 142, 1027-1042 (2012)

15. Wu, XL, Guo, B, Gao, WJ: Blow-up of solutions for a semilinear parabolic equation involving variable source and positive initial energy. Appl. Math. Lett. 26, 539-543 (2013)

16. Wang, $\mathrm{H}, \mathrm{He}, \mathrm{YJ}$ : On blow-up of solutions for a semilinear parabolic equation involving variable source and positive initial energy. Appl. Math. Lett. 26, 1008-1012 (2013)

17. Payne, L, Sattinger, O: Saddle points and instability on nonlinear hyperbolic equations. Isr. J. Math. 22, 273-303 (1975) 
18. $\mathrm{Xu}, \mathrm{RZ}, \mathrm{Su}, \mathrm{J}$ : Global existence and finite time blow-up for a class of semilinear pseudo-parabolic equations. J. Funct. Anal. 264, 2732-2763 (2013)

19. Liu, YC, Zhao, JS: On potential wells and applications to semilinear hyperbolic equations and parabolic equations. Nonlinear Anal. 64, 2665-2687 (2006)

20. Liu, BY, Ma, L: Blow up threshold for the Gross-Pitaevskii system with combined nonlocal nonlinearities. J. Math. Anal. Appl. 425, 1214-1224 (2015)

21. Liu, BY, Ma, L: Invariant sets and the blow up threshold for a nonlocal equation of parabolic type. Nonlinear Anal. 110 141-156 (2014)

22. Hoshino, H, Yamada, Y: Solvability and smoothing effect for semilinear parabolic equations. Funkc. Ekvacioj 34, 475-494 (1991)

23. Kaplan, S: On the growth of solutions of quasi-linear parabolic equations. Commun. Pure Appl. Math. 16, 305-330 (1963)

Submit your manuscript to a SpringerOpen ${ }^{\odot}$ journal and benefit from:

- Convenient online submission

Rigorous peer review

- Immediate publication on acceptance

Open access: articles freely available online

- High visibility within the field

- Retaining the copyright to your article 